\title{
Mismatch repair gene mutation spectrum in the Swedish Lynch syndrome population
}

\author{
KRISTINA LAGERSTEDT-ROBINSON ${ }^{1}$, ANNA ROHLIN ${ }^{2,3}$, CHRISTOS ARAVIDIS ${ }^{4}$, BEATRICE MELIN ${ }^{5}$, \\ MARGARETA NORDLING ${ }^{2,3}$, MARIE STENMARK-ASKMALM $^{6,9}$, ANNIKA LINDBLOM $^{1}$ and MEF NILBERT \\ ${ }^{1}$ Department of Molecular Medicine and Surgery, Karolinska Institute and Department of Clinical Genetics, \\ Karolinska University Hospital, Solna, SE-17176 Stockholm; ${ }^{2}$ Department of Clinical Pathology and Genetics, \\ Sahlgrenska University Hospital, SE-41345 Gothenburg; ${ }^{3}$ Department of Clinical Genetics, Institute of Biomedicine, \\ Sahlgrenska Academy at the University of Gothenburg, SE-40530 Gothenburg; ${ }^{4}$ Department of Immunology, \\ Genetics and Pathology, Uppsala University, SE-75185 Uppsala; ${ }^{5}$ Department of Radiation Sciences, Division of Oncology, \\ Umeå University, SE-90187 Umeå; ${ }^{6}$ Department of Oncology, Linköping University, SE-58183 Linköping; \\ ${ }^{7}$ Department of Clinical Sciences, Division of Oncology and Pathology, Lund University, SE-22381 Lund, Sweden; \\ ${ }^{8}$ Clinical Research Centre, Hvidovre Hospital, Copenhagen University, DK-2650 Hvidovre, Denmark
}

Received April 15, 2016; Accepted May 31, 2016

DOI: $10.3892 /$ or.2016.5060

\begin{abstract}
Lynch syndrome caused by constitutional mismatch-repair defects is one of the most common hereditary cancer syndromes with a high risk for colorectal, endometrial, ovarian and urothelial cancer. Lynch syndrome is caused by mutations in the mismatch repair (MMR) genes i.e., $M L H 1$, MSH2, MSH6 and PMS2. After 20 years of genetic counseling and genetic testing for Lynch syndrome, we have compiled the mutation spectrum in Sweden with the aim to provide a population-based perspective on the contribution from the different MMR genes, the various types of mutations and the influence from founder mutations. Mutation data were collected on a national basis from all laboratories involved in genetic testing. Mutation analyses were performed using mainly Sanger sequencing and multiplex ligation-dependent probe amplification. A total of 201 unique disease-predisposing MMR gene mutations were identified in 369 Lynch syndrome families. These mutations affected $M L H 1$ in $40 \%$, MSH2 in 36\%, MSH6 in $18 \%$ and PMS2 in $6 \%$ of the families. A large variety of mutations were identified with splice site mutations being the most common mutation type in $\mathrm{MLHI}$ and frameshift mutations predominating in $\mathrm{MSH} 2$ and $\mathrm{MSH}$.
\end{abstract}

Correspondence to: Dr Kristina Lagerstedt-Robinson, Department of Clinical Genetics L5:03, Karolinska University Hospital, Solna, SE-17176 Stockholm, Sweden

E-mail: kristina.lagerstedt@ki.se

Present address: ${ }^{9}$ Department of Clinical Genetics, Lund University Hospital, SE-22185 Lund, Sweden

Key words: HNPCC, MLH1, MSH2, MSH6, EPCAM, hereditary colorectal cancer, Lynch syndrome
Large deletions of one or several exons accounted for $21 \%$ of the mutations in $M L H 1$ and $M S H 2$ and $22 \%$ in $P M S 2$, but were rare (4\%) in MSH6. In $66 \%$ of the Lynch syndrome families the variants identified were private and the effect from founder mutations was limited and predominantly related to a Finnish founder mutation that accounted for $15 \%$ of the families with mutations in MLHI. In conclusion, the Swedish Lynch syndrome mutation spectrum is diverse with private MMR gene mutations in two-thirds of the families, has a significant contribution from internationally recognized mutations and a limited effect from founder mutations.

\section{Introduction}

A growing number of disease-predisposing genes are identified and contribute to the complex hereditary colorectal cancer landscape (1). An identifiable cause of cancer predisposition can be demonstrated in 5\% of colorectal cancer. Lynch syndrome is the most common hereditary colorectal cancer subtype with an estimated incidence of 1/1,200-1/660 (2). Germline mismatch-repair (MMR) gene mutations give rise to two phenotypic syndromes, i.e., the autosomal dominant, adult-onset Lynch syndrome and the recessive, childhood-onset constitutional mismatch repair deficiency (CMMRD) syndrome (3). Worldwide, more than 1,300 disease-predisposing MMR gene sequence variants have been reported (4). The estimated contribution from the different MMR genes to Lynch syndrome is $\sim 50 \%$ MLH1, 40\% MSH2 (5), 7-20\% MSH6 (5-8) and <5\% PMS2 (9). Mutations in the EPCAM gene, located upstream of $M S H 2$, represent an additional cause that is estimated to contribute to $1-3 \%$ of the disease-predisposing mutations (10-12). Founder effects, i.e., mutations that are overrepresented within a geographically or ethically isolated population, have been described in several populations, such as in the different Scandinavian populations $(8,13,14)$ and in the Ashkenazi Jewish population (15-18). 
Lynch syndrome is a multi-tumor syndrome and although the highest risks of cancer apply to colorectal, endometrial, ovarian and urinary tract cancer, a number of less common tumor types, such as cancer of the small bowel, brain tumors and skin tumors, have been linked to the syndrome (19). Different disease characteristics have been ascribed to mutations in the different MMR genes with a predominance of colorectal cancer in $M L H 1$ and $P M S 2$ mutation carriers, a high risk of extracolonic cancer in $\mathrm{MSH} 2$ mutation carriers and a high risk of gynecologic cancer in $\mathrm{MSH6}$ mutation carriers. Compared to $\mathrm{MLH1}$ and $\mathrm{MSH} 2$ mutation carriers, a later age at onset and a reduced penetrance has been described in MSH6 $(20,21)$ as well as in PMS2 mutation carriers $(9,22)$. The overall life-time risk of cancer at age 70 is estimated to be $70 \%$ (23). Age at onset is on average 20 years earlier than sporadic tumors, although the different tumor types show characteristic peak ages and phenotypes are highly variable, also within Lynch syndrome families. Identification of individuals and families with Lynch syndrome is challenging since family history has suboptimal sensitivity and the syndrome includes a broad tumor spectrum and variable penetrance and age at onset. However, reflex testing for MMR status is increasingly applied in colorectal cancer and is also discussed for endometrial cancer and will increase the likelihood of identifying individuals at increased risk in the future (24).

After 20 years of molecular diagnostics for Lynch syndrome, we compiled mutation data from the Swedish Lynch syndrome population with the aim to define the mutation spectrum, clarify the contribution from the different MMR genes, identify potential founder mutations and contribute to the world-wide data on Lynch syndrome mutations.

\section{Patients and methods}

In Sweden, general guidelines for referral of cases with suspected hereditary colorectal cancer to genetic counseling include families/individuals with three or more cases of colorectal cancer or other Lynch syndrome-associated tumors with one family member diagnosed before the age of 50 (in line with the Amsterdam criteria except for the requirement of two first-degree relatives) or a single case of colorectal cancer diagnosed before the age of 50. In addition, clinicians have referred families suspected of Lynch syndrome based on the development of Lynch syndrome-associated tumor types. Reflex testing for MMR defects in colorectal cancer has not been implemented in Swedish pathology laboratories. Targeted analysis for MMR status, typically using four-protein immunohistochemical MMR staining and/or analysis for microsatellite instability (MSI) were applied for pre-screening in most cases.

All individuals/families genetically tested and found to carry MMR gene alterations classified as disease-predisposing genetic variants or a variant of uncertain significance between January 1994 and December 2014 were eligible for the study. Mutation data were collected from the six laboratories and/or oncogenetic clinics at the University hospitals in Umeå, Uppsala, Stockholm, Linköping, Gothenburg and Lund, responsible for genetic diagnostics. The Ethics Committee at Karolinska Institutet approved the study, which followed the tenets of the Declaration of Helsinki. All patients provided oral or written informed consent for genetic diagnostics.
Genetic screening of the proband/affected family member was performed using mainly Sanger DNA sequencing or massive parallel sequencing and the analyses were combined with multiplex ligation-dependent probe amplification (MLPA, P003 and P072; MRC-Holland, Amsterdam, The Netherlands) for the detection of large deletions or duplications.

All variants reported were classified at the nucleotide and protein levels according to the Human Genome Variation Society (HGVS) nomenclature (25). As reference sequences NM_000249, NM_000251, NM_000179 and NM_000535 were used. All sequence variants were then adjusted to the classification used in the InSiGHT database (http://insight-group. org/variants/database/). Variants previously not described in the InSiGHT database were, whenever possible, classified according to the InSiGHT VIC rules (4). Frequency data for certain variants were obtained from the ExAc database using the Alamut software (Alamut Visual, v. 2.7, Interactive Biosoftware, Rouen, France). Variants with a classification of 1 (benign) or 2 (likely benign) are not included (4).

\section{Results}

In Sweden, the Lynch syndrome cohort consisted of 369 families with disease-predisposing mutations. These families were found to carry mutations in MLH1 ( $\mathrm{n}=149), M S H 2 \quad(\mathrm{n}=132$, including one family with a deletion of the EPCAM gene), MSH6 $(\mathrm{n}=67)$ and PMS2 $(\mathrm{n}=21)$ (Table I). The contributions from the different MMR genes were $M L H 1$ 40\%, MSH2 36\%, MSH6 18\% and PMS2 6\% (Fig. 1A). In total, 201 unique alterations were identified, including 48 missense sequence variants, 31 nonsense variants, 43 insertions/deletions, 35 splice site variants and 36 whole exon/exons deletions/duplications. Splice site alterations were the most common mutation type in $M L H 1$, frameshift mutations predominated in $M S H 2$ and MSH6 and missense variants were most frequent in PMS2 (Fig. 1B). Copy number variations, i.e., deletions or duplications of whole exon/exons, constituted $21 \%$ of the mutations in $M L H 1,22 \%$ in $M S H 2$ including EPCAM, $4 \%$ in $M S H 6$ and $22 \%$ in PMS2 (Fig. 1B).

The Swedish Lynch syndrome sequence variant spectrum is broad with 133 of the $201(66 \%)$ alterations being private, i.e., observed in a single family, $26 \%$ observed in 2-3 families, and 18 variants observed in $\geq 4$ families (Table II). In relation to the different genes, private mutations accounted for $46 / 71$ $M L H 1$ variants, $49 / 76 M S H 2$ variants (including the EPCAM deletion), 31/45 MSH6 variants and 6/9 PMS2 variants. Of the 201 unique variants, 137 were present in the InSiGHT LOVD with a classification made by an expert panel for 136 of these variants (4) (http://insight-group.org/variants/database/). For the remaining 64 sequence variants, 31 could, based on the predicted protein consequence from the sequence alteration, be classified as class 3-5 according to the five tier system (4).

Alterations observed in 4 or more families (Table II), i.e., recurrent alterations, included the $M L H 1$ sequence variations c.62C $>\mathrm{T}$, c. $131 \mathrm{C}>\mathrm{T}$, deletion of exon 6 (c.454-?_545+?del), c.546-2A $>$ G, deletion of exon 11 (c.885-?_1038+?del), deletion of exon 16 (c.1732-?_1896+?del) and the c.2059C $>$ T variation. These variants have previously been recognized in Lynch syndrome families and are classified as disease-predisposing. In $M S H 2$, recurrent alterations included deletion of exons 1-6 
Table I. List of sequence variants in Swedish families with Lynch syndrome.

\begin{tabular}{|c|c|c|c|c|c|c|}
\hline $\begin{array}{l}\text { Variant } \\
\text { no. }\end{array}$ & Gene & $\begin{array}{l}\text { Sequence } \\
\text { variant }\end{array}$ & $\begin{array}{l}\text { Type of variant/ } \\
\text { comment }\end{array}$ & $\begin{array}{l}\text { Change at } \\
\text { protein level }\end{array}$ & $\begin{array}{c}\text { InSiGHT } \\
\text { classification }\end{array}$ & Refs. \\
\hline 1 & $M L H 1$ & c. $-7 \mathrm{C}>\mathrm{T}$ & Other & & Class 3 & \\
\hline 2 & MLH1 & c.1-?_306+?del & Deletion exons 1-3 & & Class 5 & \\
\hline 3 & $M L H 1$ & c.1-?_306+?del & Deletion exons 1-3 & & Class 5 & \\
\hline 4 & MLH1 & c.1-?_306+?del & Deletion exons 1-3 & & Class 5 & \\
\hline 5 & MLH1 & c.1-?_1731+?del & Deletion exons 1-15 & & Class 5 & $(26)$ \\
\hline 6 & MLHI & c.1-?_2271+?del & Whole gene deletion & & Class 5 & \\
\hline 7 & MLHI & c.1-?_2271+?del & Whole gene deletion & & Class 5 & \\
\hline 8 & MLHI & c.1-?_2271+?del & Whole gene deletion & & Class 5 & \\
\hline 9 & MLH1 & c. $19 \mathrm{G}>\mathrm{T}$ & Missense & p.(Val7Phe) & & \\
\hline 10 & MLH1 & c. $62 \mathrm{C}>\mathrm{T}$ & Missense & p.(Ala21Val) & Class 4 & (27) \\
\hline 11 & MLH1 & c. $62 \mathrm{C}>\mathrm{T}$ & Missense & p.(Ala21 Val) & Class 4 & \\
\hline 12 & MLHI & c. $62 \mathrm{C}>\mathrm{T}$ & Missense & p.(Ala21 Val) & Class 4 & \\
\hline 13 & MLH1 & c. $62 \mathrm{C}>\mathrm{T}$ & Missense & p.(Ala21 Val) & Class 4 & \\
\hline 14 & MLH1 & c. $104 \mathrm{~T}>\mathrm{G}$ & Missense & p.(Met35Arg) & Class 5 & $(26)$ \\
\hline 15 & MLH1 & c.117-?_207+?del & Deletion exon 2 & p.(Cys39*) & Class 5 & \\
\hline 16 & MLHI & c.117-?_207+?del & Deletion exon 2 & p.(Cys39*) & Class 5 & \\
\hline 17 & $M L H 1$ & c.117-?_207+?del & Deletion exon 2 & p.(Cys39*) & Class 5 & \\
\hline 18 & MLH1 & c. $131 \mathrm{C}>\mathrm{T}$ & Missense & p.(Ser44Phe) & Class 5 & (26) \\
\hline 19 & MLHI & c. $131 \mathrm{C}>\mathrm{T}$ & Missense & p.(Ser44Phe) & Class 5 & (26) \\
\hline 20 & MLH1 & c. $131 \mathrm{C}>\mathrm{T}$ & Missense & p.(Ser44Phe) & Class 5 & (26) \\
\hline 21 & MLHI & c. $131 \mathrm{C}>\mathrm{T}$ & Missense & p.(Ser44Phe) & Class 5 & \\
\hline 22 & $M L H 1$ & c. $131 \mathrm{C}>\mathrm{T}$ & Missense & p.(Ser44Phe) & Class 5 & \\
\hline 23 & MLH1 & c. $199 \mathrm{G}>\mathrm{A}$ & Missense & p.(Gly67Arg) & Class 5 & (26) \\
\hline 24 & $M L H 1$ & c.202dup & Frameshift & p.(Ile68Asnfs*11) & (Class 5) & \\
\hline 25 & MLHI & c. $203 \mathrm{~T}>\mathrm{A}$ & Missense & p.(Ile68Asn) & Class 4 & $(27)$ \\
\hline 26 & MLH1 & c. $203 \mathrm{~T}>\mathrm{A}$ & Missense & p.(Ile68Asn) & Class 4 & \\
\hline 27 & $M L H 1$ & c. $208-1 \mathrm{G}>\mathrm{A}$ & Aberrant splicing & & Class 5 & \\
\hline 28 & $M L H 1$ & c. $208-2 \mathrm{~A}>\mathrm{G}$ & Aberrant splicing & & Class 5 & (26) \\
\hline 29 & MLH1 & c. $298 \mathrm{C}>\mathrm{T}$ & Nonsense & p. $(\operatorname{Arg} 100 *)$ & Class 5 & (26) \\
\hline 30 & $M L H 1$ & c. $306+1 \mathrm{G}>\mathrm{A}$ & Aberrant splicing & p.(Lys70_Glu102del) & Class 4 & \\
\hline 31 & MLH1 & c. $306+1 \mathrm{G}>\mathrm{A}$ & Aberrant splicing & p.(Lys70_Glu102del) & Class 4 & \\
\hline 32 & $M L H 1$ & c. $306+3 \mathrm{~A}>\mathrm{C}$ & Aberrant splicing & & Class 3 & $(26)$ \\
\hline 33 & $M L H 1$ & c.307-?_1038+?del & Deletion exons 4-11 & p.(Ala103Argfs*8) & Class 5 & $(26)$ \\
\hline 34 & $M L H 1$ & c.307-?_545+?del & Deletion exons 4-6 & p.(Ala103Valfs*9) & Class 5 & \\
\hline 35 & $M L H 1$ & c.307-?_677+?del & Deletion exons 4-8 & & (Class 5) & \\
\hline 36 & MLH1 & c. $34 \mathrm{G}>\mathrm{T}$ & Missense & p.(Asp12Tyr) & & \\
\hline 37 & MLH1 & c. $350 \mathrm{C}>\mathrm{T}$ & Missense & p.(Thr117Met) & Class 5 & \\
\hline 38 & MLH1 & c. $350 \mathrm{C}>\mathrm{T}$ & Missense & p.(Thr117Met) & Class 5 & \\
\hline 39 & $M L H 1$ & c. $409 \mathrm{G}>\mathrm{A}$ & Missense & p.(Ala137Thr) & & \\
\hline 40 & $M L H 1$ & c. $409 \mathrm{G}>\mathrm{A}$ & Missense & p.(Ala137Thr) & & \\
\hline 41 & MLH1 & c.454-?_545+?del & Deletion exon 6 & p.(Glu153Phefs*8) & Class 5 & \\
\hline 42 & MLHI & c.454-?_545+?del & Deletion exon 6 & p.(Glu153Phefs*8) & Class 5 & \\
\hline 43 & MLHI & c.454-?_545+?del & Deletion exon 6 & p.(Glu153Phefs*8) & Class 5 & 26) \\
\hline 44 & MLH1 & c.454-?_545+?del & Deletion exon 6 & p.(Glu153Phefs*8) & Class 5 & \\
\hline 45 & MLH1 & c. $454-13 \mathrm{~A}>\mathrm{G}$ & Aberrant splicing & & Class 3 & $(26)$ \\
\hline 46 & MLH1 & c. $454-1 \mathrm{G}>\mathrm{A}$ & Aberrant splicing & p.(Glu153Phefs*8) & Class 5 & \\
\hline 47 & MLH1 & c. $454-1 \mathrm{G}>\mathrm{A}$ & Aberrant splicing & p.(Glu153Phefs*8) & Class 5 & $(26)$ \\
\hline 48 & MLHI & c. $546-2 \mathrm{~A}>\mathrm{G}$ & Aberrant splicing & p. $(\operatorname{Arg} 182 \operatorname{Serfs} * 6)$ & Class 5 & (26) \\
\hline 49 & MLH1 & c. $546-2 \mathrm{~A}>\mathrm{G}$ & Aberrant splicing & p. $(\operatorname{Arg} 182 \operatorname{Serfs} * 6)$ & Class 5 & \\
\hline 50 & $M L H 1$ & c. $546-2 \mathrm{~A}>\mathrm{G}$ & Aberrant splicing & p. $(\operatorname{Arg} 182 \operatorname{Serfs} * 6)$ & Class 5 & \\
\hline
\end{tabular}


Table I. Continued.

\begin{tabular}{|c|c|c|c|c|c|c|}
\hline $\begin{array}{l}\text { Variant } \\
\text { no. }\end{array}$ & Gene & $\begin{array}{l}\text { Sequence } \\
\text { variant }\end{array}$ & $\begin{array}{l}\text { Type of variant/ } \\
\text { comment }\end{array}$ & $\begin{array}{c}\text { Change at } \\
\text { protein level }\end{array}$ & $\begin{array}{c}\text { InSiGHT } \\
\text { classification }\end{array}$ & Refs. \\
\hline 51 & $M L H 1$ & c. $546-2 \mathrm{~A}>\mathrm{G}$ & Aberrant splicing & p.(Arg182Serfs*6) & Class 5 & \\
\hline 52 & $M L H 1$ & c. $546-2 \mathrm{~A}>\mathrm{G}$ & Aberrant splicing & p. $(\operatorname{Arg} 182 \operatorname{Serfs} * 6)$ & Class 5 & \\
\hline 53 & $M L H 1$ & c. $546-2 \mathrm{~A}>\mathrm{G}$ & Aberrant splicing & p.(Arg182Serfs*6) & Class 5 & \\
\hline 54 & MLHI & c. $546-2 \mathrm{~A}>\mathrm{G}$ & Aberrant splicing & p. $(\operatorname{Arg} 182 \operatorname{Serfs} * 6)$ & Class 5 & \\
\hline 55 & $M L H 1$ & c. $546-2 \mathrm{~A}>\mathrm{G}$ & Aberrant splicing & p. $(\operatorname{Arg} 182 \operatorname{Serfs} * 6)$ & Class 5 & \\
\hline 56 & $M L H 1$ & c. $546-2 \mathrm{~A}>\mathrm{G}$ & Aberrant splicing & p.(Arg182Serfs*6) & Class 5 & \\
\hline 57 & $M L H 1$ & c. $546-2 \mathrm{~A}>\mathrm{G}$ & Aberrant splicing & p.(Arg 182 Serfs*6) & Class 5 & \\
\hline 58 & $M L H 1$ & c. $546-2 \mathrm{~A}>\mathrm{G}$ & Aberrant splicing & p.(Arg 182 Serfs*6) & Class 5 & \\
\hline 59 & $M L H 1$ & c. $546-2 \mathrm{~A}>\mathrm{G}$ & Aberrant splicing & p.(Arg182Serfs*6) & Class 5 & \\
\hline 60 & $M L H 1$ & c. $.588+1 \mathrm{del}$ & Aberrant splicing & & Class 4 & \\
\hline 61 & $M L H 1$ & c.589-?_790+?dup & Duplication exons 8-9 & & & \\
\hline 62 & $M L H 1$ & c.665del & Frameshift & p.(Asn222Metfs*7) & Class 5 & (26) \\
\hline 63 & $M L H 1$ & c. $665 \mathrm{del}$ & Frameshift & p.(Asn222Metfs*7) & Class 5 & \\
\hline 64 & $M L H 1$ & c. $676 \mathrm{C}>\mathrm{T}$ & Nonsense & p. $(\operatorname{Arg} 226 *)$ & Class 5 & \\
\hline 65 & $M L H 1$ & c. $677+1 \mathrm{G}>\mathrm{T}$ & Aberrant splicing & & Class 5 & (26) \\
\hline 66 & $M L H 1$ & c. $677 \mathrm{G}>\mathrm{A}$ & Aberrant splicing & p.(Gln197Argfs*8) & Class 5 & \\
\hline 67 & $M L H 1$ & c.679_689del & Frameshift & p.(Glu227Asnfs*4) & (Class 5) & \\
\hline 68 & $M L H 1$ & c. $790+1 \mathrm{G}>\mathrm{C}$ & Aberrant splicing & p.(Glu227_Ser295del) & Class 4 & \\
\hline 69 & $M L H 1$ & c. $790+1 \mathrm{G}>\mathrm{C}$ & Aberrant splicing & p.(Glu227_Ser295del) & Class 4 & \\
\hline 70 & $M L H 1$ & c. $793 \mathrm{C}>\mathrm{T}$ & Aberrant splicing & p.(His264Leufs*2) & Class 5 & (26) \\
\hline 71 & $M L H 1$ & c. $793 \mathrm{C}>\mathrm{T}$ & Aberrant splicing & p.(His264Leufs*2) & Class 5 & \\
\hline 72 & $M L H 1$ & c. $793 \mathrm{C}>\mathrm{T}$ & Aberrant splicing & p.(His264Leufs*2) & Class 5 & \\
\hline 73 & $M L H 1$ & c.885-?_1038+?del & Deletion exon 11 & p.(Ser295Argfs*21) & Class 5 & (26) \\
\hline 74 & $M L H 1$ & c.885-?_1038+?del & Deletion exon 11 & p.(Ser295Argfs*21) & Class 5 & \\
\hline 75 & $M L H 1$ & c.885-?_1038+?del & Deletion exon 11 & p.(Ser295Argfs*21) & Class 5 & \\
\hline 76 & $M L H 1$ & c.885-?_1038+?del & Deletion exon 11 & p.(Ser295Argfs*21) & Class 5 & \\
\hline 77 & $M L H 1$ & c.885-?_1409+?del & Deletion exons 11-12 & & (Class 5) & \\
\hline 78 & $M L H 1$ & c.885-?_1409+?del & Deletion exons 11-12 & & (Class 5) & \\
\hline 79 & $M L H 1$ & c. $955 \mathrm{G}>\mathrm{A}$ & Missense & p.(Glu319Lys) & Class 3 & \\
\hline 80 & $M L H 1$ & c. $958 \mathrm{G}>\mathrm{T}$ & Nonsense & p.(Glu320*) & (Class 5) & \\
\hline 81 & $M L H 1$ & c.1050del & Frameshift & p.(Gly351Aspfs*16) & Class 5 & \\
\hline 82 & $M L H 1$ & c. $1219 \mathrm{C}>\mathrm{T}$ & Nonsense & p. $(\mathrm{G} \ln 407 *)$ & (Class 5) & \\
\hline 83 & $M L H 1$ & c. $1225 \mathrm{C}>\mathrm{T}$ & Nonsense & p. $(\mathrm{G} \ln 409 *)$ & Class 5 & (26) \\
\hline 84 & $M L H 1$ & c.1309_1310del & Frameshift & p.(Pro437Cysfs*2) & (Class 5) & \\
\hline 85 & $M L H 1$ & c. $1379 \mathrm{~A}>\mathrm{C}$ & Missense & p.(Glu460Ala) & & \\
\hline 86 & $M L H 1$ & c. $1379 A>C$ & Missense & p.(Glu460Ala) & & \\
\hline 87 & $M L H 1$ & c. $1379 A>C$ & Missense & p.(Glu460Ala) & & \\
\hline 88 & $M L H 1$ & c. $1459 \mathrm{C}>\mathrm{T}$ & Nonsense & p.(Arg487*) & Class 5 & (26) \\
\hline 89 & $M L H 1$ & c.1559-?_1730+?del & Deletion exons 14-15 & p.(Val520Glyfs*7) & Class 5 & (26) \\
\hline 90 & $M L H 1$ & c.1559-?_2271+?del & Deletion exons 14-19 & & Class 5 & \\
\hline 91 & $M L H 1$ & c. $1564 \mathrm{C}>\mathrm{T}$ & Missense & p.(Arg522Trp) & & \\
\hline 92 & MLH1 & c. $1609 \mathrm{C}>\mathrm{T}$ & Nonsense & p. $(\mathrm{G} \ln 537 *)$ & Class 5 & \\
\hline 93 & MLH1 & c.1667+2_1667+8delinsATTT & Aberrant splicing & & Class 5 & \\
\hline 94 & MLH1 & c.1667+2_1667+8delinsATTT & Aberrant splicing & & Class 5 & \\
\hline 95 & $M L H 1$ & c. $1668-1 \mathrm{G}>\mathrm{T}$ & Aberrant splicing & & Class 4 & \\
\hline 96 & MLH1 & c. $1730 \mathrm{C}>\mathrm{T}$ & Missense & p.(Ser577Leu) & & \\
\hline 97 & MLH1 & c. $1730 \mathrm{C}>\mathrm{T}$ & Missense & p.(Ser577Leu) & & \\
\hline 98 & $M L H 1$ & c. $1731 \mathrm{G}>\mathrm{A}$ & Aberrant splicing & p.(Ser556Argfs*14) & Class 5 & \\
\hline 99 & $M L H 1$ & c.1732-?_1896+?del & Deletion exon 16 & p.(Pro579_Glu633del) & Class 5 & $(27)$ \\
\hline 100 & MLH1 & c.1732-?_1896+?del & Deletion exon 16 & p.(Pro579_Glu633del) & Class 5 & $(26)$ \\
\hline 101 & MLHI & c.1732-?_1896+?del & Deletion exon 16 & p.(Pro579_Glu633del) & Class 5 & (26) \\
\hline
\end{tabular}


Table I. Continued.

\begin{tabular}{|c|c|c|c|c|c|c|}
\hline $\begin{array}{l}\text { Variant } \\
\text { no. }\end{array}$ & Gene & $\begin{array}{l}\text { Sequence } \\
\text { variant }\end{array}$ & $\begin{array}{l}\text { Type of variant/ } \\
\text { comment }\end{array}$ & $\begin{array}{c}\text { Change at } \\
\text { protein level }\end{array}$ & $\begin{array}{c}\text { InSiGHT } \\
\text { classification }\end{array}$ & Refs. \\
\hline 102 & $M L H 1$ & c.1732-?_1896+?del & Deletion exon 16 & p.(Pro579_Glu633del) & Class 5 & (26) \\
\hline 103 & $M L H 1$ & c.1732-?_1896+?del & Deletion exon 16 & p.(Pro579_Glu633del) & Class 5 & (26) \\
\hline 104 & $M L H 1$ & c.1732-?_1896+?del & Deletion exon 16 & p.(Pro579_Glu633del) & Class 5 & (26) \\
\hline 105 & $M L H 1$ & c.1732-?_1896+?del & Deletion exon 16 & p.(Pro579_Glu633del) & Class 5 & (26) \\
\hline 106 & $M L H 1$ & c.1732-?_1896+?del & Deletion exon 16 & p.(Pro579_Glu633del) & Class 5 & \\
\hline 107 & $M L H 1$ & c.1732-?_1896+?del & Deletion exon 16 & p.(Pro579_Glu633del) & Class 5 & \\
\hline 108 & $M L H 1$ & c.1732-?_1896+?del & Deletion exon 16 & p.(Pro579_Glu633del) & Class 5 & \\
\hline 109 & $M L H 1$ & c.1732-?_1896+?del & Deletion exon 16 & p.(Pro579_Glu633del) & Class 5 & \\
\hline 110 & $M L H 1$ & c.1732-?_1896+?del & Deletion exon 16 & p.(Pro579_Glu633del) & Class 5 & \\
\hline 111 & $M L H 1$ & c.1732-?_1896+?del & Deletion exon 16 & p.(Pro579_Glu633del) & Class 5 & \\
\hline 112 & $M L H 1$ & c.1732-?_1896+?del & Deletion exon 16 & p.(Pro579_Glu633del) & Class 5 & (26) \\
\hline 113 & MLH1 & c.1732-?_1896+?del & Deletion exon 16 & p.(Pro579_Glu633del) & Class 5 & \\
\hline 114 & $M L H 1$ & c.1732-?_1896+?del & Deletion exon 16 & p.(Pro579_Glu633del) & Class 5 & \\
\hline 115 & $M L H 1$ & c.1732-?_1896+?del & Deletion exon 16 & p.(Pro579_Glu633del) & Class 5 & \\
\hline 116 & $M L H 1$ & c.1732-?_1896+?del & Deletion exon 16 & p.(Pro579_Glu633del) & Class 5 & \\
\hline 117 & $M L H 1$ & c.1732-?_1896+?del & Deletion exon 16 & p.(Pro579_Glu633del) & Class 5 & \\
\hline 118 & $M L H 1$ & c.1732-?_1896+?del & Deletion exon 16 & p.(Pro579_Glu633del) & Class 5 & \\
\hline 119 & $M L H 1$ & c.1732-?_1896+?del & Deletion exon 16 & p.(Pro579_Glu633del) & Class 5 & \\
\hline 120 & $M L H 1$ & c.1732-?_1896+?del & Deletion exon 16 & p.(Pro579_Glu633del) & Class 5 & \\
\hline 121 & $M L H 1$ & c.1732-?_1896+?del & Deletion exon 16 & p.(Pro579_Glu633del) & Class 5 & \\
\hline 122 & $M L H 1$ & c.1732-?_2271+?del & Deletion exons 16-19 & & Class 5 & \\
\hline 123 & $M L H 1$ & c. $1732-2 \mathrm{~A}>\mathrm{T}$ & Aberrant splicing & p.(Pro579_Glu633del) & Class 5 & \\
\hline 124 & $M L H 1$ & c.1769del & Nonsense & p.(Leu590*) & Class 5 & (26) \\
\hline 125 & $M L H 1$ & c.1769del & Nonsense & p.(Leu590*) & Class 5 & \\
\hline 126 & $M L H 1$ & c.1772_1775del & Frameshift & p.(Asp591Valfs*24) & Class 5 & (26) \\
\hline 127 & $M L H 1$ & c.1772_1775del & Frameshift & p.(Asp591Valfs*24) & Class 5 & \\
\hline 128 & $M L H 1$ & c.1812dup & Frameshift & p.(Glu605Argfs*5) & Class 5 & \\
\hline 129 & $M L H 1$ & c.1852_1854del & Other & p.(Lys618del) & Class 5 & (27) \\
\hline 130 & $M L H 1$ & c.1852_1854del & Other & p.(Lys618del) & Class 5 & \\
\hline 131 & $M L H 1$ & c. $1896+1 \mathrm{G}>\mathrm{T}$ & Aberrant splicing & & Class 4 & \\
\hline 132 & $M L H 1$ & c. $1939 \mathrm{G}>\mathrm{A}$ & Missense & p.(Val647Met) & Class 3 & \\
\hline 133 & $M L H 1$ & c. $1943 \mathrm{C}>\mathrm{T}$ & Missense & p.(Pro648Leu) & Class 5 & \\
\hline $134 *$ & MLH1 & c. $1989 \mathrm{G}>\mathrm{A}$ & Aberrant splicing & & Class 4 & \\
\hline 135 & $M L H 1$ & c. $2038 \mathrm{~T}>\mathrm{C}$ & Missense & p.(Cys680Arg) & Class 5 & \\
\hline 136 & $M L H 1$ & c. $2059 \mathrm{C}>\mathrm{T}$ & Missense & p.(Arg687Trp) & Class 5 & (26) \\
\hline 137 & $M L H 1$ & c. $2059 \mathrm{C}>\mathrm{T}$ & Missense & p.(Arg687Trp) & Class 5 & \\
\hline 138 & $M L H 1$ & c. $2059 \mathrm{C}>\mathrm{T}$ & Missense & p.(Arg687Trp) & Class 5 & \\
\hline 139 & $M L H 1$ & c. $2059 \mathrm{C}>\mathrm{T}$ & Missense & p. (Arg687Trp) & Class 5 & (26) \\
\hline 140 & $M L H 1$ & c. $2059 \mathrm{C}>\mathrm{T}$ & Missense & p. (Arg687Trp) & Class 5 & \\
\hline 141 & $M L H 1$ & c. $2059 \mathrm{C}>\mathrm{T}$ & Missense & p. $(\operatorname{Arg} 687 \operatorname{Trp})$ & Class 5 & \\
\hline 142 & MLH1 & c. $2059 \mathrm{C}>\mathrm{T}$ & Missense & p.(Arg687Trp) & Class 5 & \\
\hline 143 & $M L H 1$ & c. $2059 \mathrm{C}>\mathrm{T}$ & Missense & p.(Arg687Trp) & Class 5 & \\
\hline 144 & $M L H 1$ & c. $2059 \mathrm{C}>\mathrm{T}$ & Missense & p.(Arg687Trp) & Class 5 & \\
\hline 145 & $M L H 1$ & c. $2059 \mathrm{C}>\mathrm{T}$ & Missense & p.(Arg687Trp) & Class 5 & \\
\hline 146 & $M L H 1$ & c.2076dup & Nonsense & p.(Glu693*) & (Class 5) & \\
\hline 147 & $M L H 1$ & c. $2103+1 G>A$ & Aberrant splicing & & Class 5 & \\
\hline 148 & $M L H 1$ & c.2104-11_2104-10delinsA & Aberrant splicing & & Class 3 & $(26)$ \\
\hline 149 & $M L H 1$ & c. $2141 \mathrm{G}>\mathrm{A}$ & Nonsense & p.(Trp714*) & Class 5 & \\
\hline 150 & $M S H 2$ & c.1-?_1076+?del & Deletion exons 1-6 & & Class 5 & \\
\hline 151 & MSH2 & c.1-?_1076+?del & Deletion exons 1-6 & & Class 5 & \\
\hline 152 & MSH2 & c.1-?_1076+?del & Deletion exons 1-6 & & Class 5 & \\
\hline
\end{tabular}


Table I. Continued.

\begin{tabular}{|c|c|c|c|c|c|c|}
\hline $\begin{array}{l}\text { Variant } \\
\text { no. }\end{array}$ & Gene & $\begin{array}{l}\text { Sequence } \\
\text { variant }\end{array}$ & $\begin{array}{l}\text { Type of variant/ } \\
\text { comment }\end{array}$ & $\begin{array}{l}\text { Change at } \\
\text { protein level }\end{array}$ & $\begin{array}{c}\text { InSiGHT } \\
\text { classification }\end{array}$ & Refs. \\
\hline 153 & MSH2 & c.1-?_1076+?del & Deletion exons 1-6 & & Class 5 & \\
\hline 154 & MSH2 & c.1-?_1076+?del & Deletion exons 1-6 & & Class 5 & $(26)$ \\
\hline 155 & MSH2 & c.1-?_1076+?del & Deletion exons 1-6 & & Class 5 & (26) \\
\hline 156 & MSH2 & c.1-?_1076+?del & Deletion exons 1-6 & & Class 5 & \\
\hline 157 & MSH2 & c.1-?_1076+?del & Deletion exons 1-6 & & Class 5 & \\
\hline 158 & MSH2 & c.1-?_1076+?del & Deletion exons 1-6 & & Class 5 & \\
\hline 159 & MSH2 & c.1-?_1076+?del & Deletion exons 1-6 & & Class 5 & \\
\hline 160 & MSH2 & c.1-?_1276+?del & Deletion exons 1-7 & & Class 5 & $(26)$ \\
\hline 161 & MSH2 & c.1-?_1276+?del & Deletion exons 1-7 & & Class 5 & \\
\hline 162 & MSH2 & c.1-?_1386+?del & Deletion exons $1-8$ & & Class 5 & \\
\hline 163 & MSH2 & c.1-?_1386+?del & Deletion exons 1-8 & & Class 5 & $(26)$ \\
\hline 164 & MSH2 & c.1-?_1386+?del & Deletion exons 1-8 & & Class 5 & \\
\hline 165 & MSH2 & c.1-?_1386+?del & Deletion exons 1-8 & & Class 5 & \\
\hline 166 & MSH2 & c.1-?_1386+?del & Deletion exons $1-8$ & & Class 5 & \\
\hline 167 & MSH2 & c.1-?_1386+?del & Deletion exons 1-8 & & Class 5 & \\
\hline 168 & MSH2 & c.1-?_1386+?del & Deletion exons 1-8 & & Class 5 & \\
\hline 169 & MSH2 & c.1-?_1661+?del & Deletion exons 1-11 & & Class 5 & \\
\hline 170 & $M S H 2$ & c.17_20del & Frameshift & p.(Lys6Argfs*57) & (Class 5) & \\
\hline 171 & MSH2 & c. $138 \mathrm{C}>\mathrm{G}$ & Missense & p.(His46Gln) & Class 3 & \\
\hline 172 & MSH2 & c. $183 \mathrm{G}>\mathrm{T}$ & Missense & p.(Gln61His) & & \\
\hline 173 & $M S H 2$ & c.187del & Nonsense & p. $(\operatorname{Val} 63 *)$ & Class 5 & \\
\hline 174 & MSH2 & c.204del & Frameshift & p.(Pro69Argfs*15) & Class 5 & $(26)$ \\
\hline 175 & MSH2 & c.212-?_366+?del & Deletion exon 2 & p.(Ala72Phefs*9) & Class 5 & (26) \\
\hline 176 & MSH2 & c.212-?_366+?del & Deletion exon 2 & p.(Ala72Phefs*9) & Class 5 & \\
\hline 177 & MSH2 & c.212-?_1276+?del & Deletion exons 2-7 & p.(Ala72_Gly426del) & Class 5 & \\
\hline 178 & $M S H 2$ & c. $366+1 \mathrm{G}>\mathrm{C}$ & Aberrant splicing & p.(Ala72Phefs*9) & Class 4 & \\
\hline 179 & MSH2 & c.416delA & Frameshift & p.(Asn139Metfs*35) & Class 5 & \\
\hline 180 & $M S H 2$ & c. $499 \mathrm{G}>\mathrm{C}$ & Missense & p.(Asp167His) & Class 3 & \\
\hline 181 & $M S H 2$ & c. $.508 \mathrm{C}>\mathrm{T}$ & Nonsense & p.(Gln170*) & Class 5 & \\
\hline 182 & $M S H 2$ & c.518_519del & Frameshift & p.(Leu173Argfs*4) & (Class 5) & \\
\hline 183 & MSH2 & c. $.557 \mathrm{~A}>\mathrm{G}$ & Missense & p.(Asn186Ser) & Class 3 & \\
\hline 184 & $M S H 2$ & c.646-?_1076+?del & Deletion exons 4-6 & p.(Ile217Glufs*28) & Class 5 & \\
\hline 185 & MSH2 & c.646-?_1076+?del & Deletion exons 4-6 & p.(Ile217Glufs*28) & Class 5 & \\
\hline 186 & MSH2 & c. $646-1 \mathrm{G}>\mathrm{A}$ & Aberrant splicing & & & \\
\hline 187 & MSH2 & c.793-?_1076+?del & Deletion exons 5-6 & p.(Val265Ilefs*29) & Class 5 & \\
\hline 188 & MSH2 & c. $793-1 \mathrm{G}>\mathrm{A}$ & Aberrant splicing & & & \\
\hline 189 & MSH2 & c.811_814del & Frameshift & p.(Ser271Argfs*2) & Class 5 & $(26)$ \\
\hline 190 & MSH2 & c. $892 \mathrm{C}>\mathrm{T}$ & Nonsense & p. $\left(\mathrm{Gln} 298^{*}\right)$ & Class 5 & $(26)$ \\
\hline 191 & MSH2 & c. $942+1 G>T$ & Aberrant splicing & & Class 4 & \\
\hline 192 & MSH2 & c. $942+3 \mathrm{~A}>\mathrm{T}$ & Aberrant splicing & p.(Val265_Gln314del) & Class 5 & $(26)$ \\
\hline 193 & $M S H 2$ & c. $942+3 \mathrm{~A}>\mathrm{T}$ & Aberrant splicing & p.(Val265_Gln314del) & Class 5 & $(26)$ \\
\hline 194 & MSH2 & c. $942+3 \mathrm{~A}>\mathrm{T}$ & Aberrant splicing & p.(Val265_Gln314del) & Class 5 & \\
\hline 195 & MSH2 & c. $942+3 \mathrm{~A}>\mathrm{T}$ & Aberrant splicing & p.(Val265_Gln314del) & Class 5 & \\
\hline 196 & MSH2 & c. $942+3 \mathrm{~A}>\mathrm{T}$ & Aberrant splicing & p.(Val265_Gln314del) & Class 5 & \\
\hline 197 & MSH2 & c. $942+3 \mathrm{~A}>\mathrm{T}$ & Aberrant splicing & p.(Val265_Gln314del) & Class 5 & \\
\hline 198 & MSH2 & c. $942+3 \mathrm{~A}>\mathrm{T}$ & Aberrant splicing & p.(Val265_Gln314del) & Class 5 & \\
\hline 199 & MSH2 & c. $942 \mathrm{G}>\mathrm{A}$ & Aberrant splicing & p.(Val265_Gln314del) & Class 5 & \\
\hline 200 & MSH2 & c.989T>C & Missense & p.(Leu330Pro) & Class 4 & \\
\hline 201 & MSH2 & c. $997 \mathrm{~T}>\mathrm{A}$ & Missense & p.(Cys333Ser) & & \\
\hline 202 & MSH2 & c.1077-?_1276+?del & Deletion exon 7 & p.(Leu360Lysfs*16) & Class 5 & \\
\hline 203 & MSH2 & c.1077-?_1276+?del & Deletion exon 7 & p.(Leu360Lysfs*16) & Class 5 & \\
\hline
\end{tabular}


Table I. Continued.

\begin{tabular}{|c|c|c|c|c|c|c|}
\hline $\begin{array}{l}\text { Variant } \\
\text { no. }\end{array}$ & Gene & $\begin{array}{l}\text { Sequence } \\
\text { variant }\end{array}$ & $\begin{array}{l}\text { Type of variant/ } \\
\text { comment }\end{array}$ & $\begin{array}{l}\text { Change at } \\
\text { protein level }\end{array}$ & $\begin{array}{c}\text { InSiGHT } \\
\text { classification }\end{array}$ & Refs. \\
\hline 204 & MSH2 & c.1077-?_1386+?dup & Duplication exons 7-8 & & Class 3 & (26) \\
\hline 205 & MSH2 & c.1077-?_1386+?dup & Duplication exons $7-8$ & & Class 3 & \\
\hline 206 & MSH2 & c.1077-?_1661+?del & Deletion exons 7-10 & p.(Arg359_Asn553del) & Class 5 & $(27)$ \\
\hline 207 & MSH2 & c.1077-?_1661+?del & Deletion exons 7-10 & p.(Arg359_Asn553del) & Class 5 & \\
\hline 208 & MSH2 & c.1077-?_1661+?del & Deletion exons 7-10 & p.(Arg359_Asn553del) & Class 5 & \\
\hline 209 & MSH2 & c.1077-?_1661+?del & Deletion exons 7-10 & p.(Arg359_Asn553del) & Class 5 & \\
\hline 210 & MSH2 & c.1077-?_1661+?del & Deletion exons 7-10 & p.(Arg359_Asn553del) & Class 5 & \\
\hline 211 & MSH2 & c.1077-?_1661+?del & Deletion exons 7-10 & p.(Arg359_Asn553del) & Class 5 & \\
\hline 212 & MSH2 & c. $1077-1 \mathrm{G}>\mathrm{A}$ & Aberrant splicing & & & \\
\hline 213 & MSH2 & c. $1077-1 \mathrm{G}>\mathrm{A}$ & Aberrant splicing & & & \\
\hline 214 & MSH2 & c.1097_1098insA & Frameshift & p.(Phe366Leufs*23) & Class 5 & \\
\hline 215 & MSH2 & c.1097_1098insA & Frameshift & p.(Phe366Leufs*23) & Class 5 & (26) \\
\hline 216 & MSH2 & c.1097_1098insA & Frameshift & p.(Phe366Leufs*23) & Class 5 & \\
\hline 217 & MSH2 & c. $1147 \mathrm{C}>\mathrm{T}$ & Nonsense & p.(Arg383*) & Class 5 & \\
\hline 218 & MSH2 & c. $1147 \mathrm{C}>\mathrm{T}$ & Nonsense & p.(Arg383*) & Class 5 & \\
\hline 219 & MSH2 & c.1162-?_2805+?del & Deletion exons 11-16 & & (Class 5) & \\
\hline 220 & MSH2 & c. $1164 \mathrm{C}>\mathrm{G}$ & Missense & p.(Asn388Lys) & & \\
\hline 221 & MSH2 & c. $1165 \mathrm{C}>\mathrm{T}$ & Nonsense & p. $(\operatorname{Arg} 389 *)$ & Class 5 & \\
\hline 222 & MSH2 & c. $1165 \mathrm{C}>\mathrm{T}$ & Nonsense & p.(Arg389*) & Class 5 & \\
\hline 223 & $M S H 2$ & c.1204del & Frameshift & p.(Gln402Lysfs*10) & Class 5 & \\
\hline 224 & MSH2 & c.1204del & Frameshift & p.(Gln402Lysfs*10) & Class 5 & \\
\hline 225 & MSH2 & c. $1216 \mathrm{C}>\mathrm{T}$ & Nonsense & p. $\left(\operatorname{Arg} 406^{*}\right)$ & Class 5 & (26) \\
\hline 226 & $M S H 2$ & c. $1225 \mathrm{C}>\mathrm{T}$ & Nonsense & p. $(\mathrm{G} \ln 409 *)$ & (Class 5) & \\
\hline 227 & MSH2 & c.1226_1227del & Frameshift & p.(Gln409Argfs*7) & Class 5 & (26) \\
\hline 228 & MSH2 & c.1237del & Frameshift & p.(Gln413Asnfs*25) & (Class 5) & \\
\hline 229 & MSH2 & c. $1275 \mathrm{~A}>\mathrm{G}$ & Aberrant splicing & p. $(=$, Ile411_Gly426del $)$ & Class 3 & \\
\hline 230 & MSH2 & c.1277-?_1386+?del & Deletion exon 8 & p.(Lys427Glyfs*4) & Class 5 & (26) \\
\hline 231 & $\mathrm{MSH} 2$ & c.1277-?_1386+?del & Deletion exon 8 & p.(Lys427Glyfs*4) & Class 5 & \\
\hline 232 & MSH2 & c. $1373 \mathrm{~T}>\mathrm{G}$ & Nonsense & p.(Leu458*) & Class 5 & $(26)$ \\
\hline 233 & MSH2 & c.1387-?_1661+?del & Deletion exons 9-10 & p.(Val463Glnfs*7) & Class 5 & \\
\hline 234 & $M S H 2$ & c.1447_1448del & Frameshift & p.(Glu483Asnfs*4) & Class 5 & $(26)$ \\
\hline 235 & MSH2 & c.1447_1448del & Frameshift & p.(Glu483Asnfs*4) & Class 5 & \\
\hline 236 & $\mathrm{MSH} 2$ & c. $1447 \mathrm{G}>\mathrm{T}$ & Nonsense & p.(Glu483*) & Class 5 & $(26)$ \\
\hline 237 & $M S H 2$ & c. $1447 \mathrm{G}>\mathrm{T}$ & Nonsense & p.(Glu483*) & Class 5 & \\
\hline $238 * *$ & MSH2 & c. $1484 \mathrm{C}>\mathrm{T}$ & Missense & p.(Thr495Ile) & & \\
\hline 239 & MSH2 & c.1490_1492del & Other & p.(Ile497del) & & \\
\hline 240 & MSH2 & c.1520del & Frameshift & p.(Pro507Leufs*19) & (Class 5) & \\
\hline 241 & MSH2 & c.1587del & Frameshift & p.(Glu530Lysfs*13) & Class 5 & \\
\hline 242 & MSH2 & c.1587del & Frameshift & p.(Glu530Lysfs*13) & Class 5 & \\
\hline 243 & MSH2 & c.1587del & Frameshift & p.(Glu530Lysfs*13) & Class 5 & \\
\hline 244 & $\mathrm{MSH} 2$ & c. $1661+5 \mathrm{G}>\mathrm{C}$ & Aberrant splicing & p.(Gly504Alafs*3) & Class 3 & \\
\hline 245 & $\mathrm{MSH} 2$ & c.1662-?_2805+?del & Deletion exons 11-16 & & Class 5 & \\
\hline 246 & MSH2 & c. $1703 \mathrm{C}>\mathrm{G}$ & Missense & p.(Thr568Arg) & & \\
\hline 247 & MSH2 & c. $1759 \mathrm{G}>\mathrm{C}$ & Aberrant splicing & p.(Ser554Argfs*11) & Class 5 & \\
\hline 248 & MSH2 & c. $1777 \mathrm{C}>\mathrm{T}$ & Nonsense & p.(Gln593*) & Class 5 & \\
\hline 249 & MSH2 & c. $1777 \mathrm{C}>\mathrm{T}$ & Nonsense & p. $\left(\mathrm{G} \ln 593^{*}\right)$ & Class 5 & \\
\hline 250 & MSH2 & c.1786_1788del & Other & p.(Asn596del) & Class 5 & \\
\hline 251 & MSH2 & c.1786_1788del & Other & p.(Asn596del) & Class 5 & \\
\hline 252 & MSH2 & c.1786_1788del & Other & p.(Asn596del) & Class 5 & \\
\hline 253 & MSH2 & c.1786_1788del & Other & p.(Asn596del) & Class 5 & \\
\hline 254 & MSH2 & c.1786_1788del & Other & p.(Asn596del) & Class 5 & \\
\hline
\end{tabular}


Table I. Continued.

\begin{tabular}{|c|c|c|c|c|c|c|}
\hline $\begin{array}{l}\text { Variant } \\
\text { no. }\end{array}$ & Gene & $\begin{array}{l}\text { Sequence } \\
\text { variant }\end{array}$ & $\begin{array}{l}\text { Type of variant/ } \\
\text { comment }\end{array}$ & $\begin{array}{l}\text { Change at } \\
\text { protein level }\end{array}$ & $\begin{array}{c}\text { InSiGHT } \\
\text { classification }\end{array}$ & Refs. \\
\hline 255 & $M S H 2$ & c.1786_1788del & Other & p.(Asn596del) & Class 5 & \\
\hline 256 & MSH2 & c. $1807 \mathrm{G}>\mathrm{A}$ & Missense & p.(Asp603Asn) & Class 3 & \\
\hline 257 & $M S H 2$ & c.1858_1859dup & Frameshift & p.(Arg621Tyrfs*15) & Class 5 & \\
\hline 258 & MSH2 & c.1881dup & Frameshift & p.(Gly628Argfs*16) & (Class 5) & \\
\hline 259 & MSH2 & c. $1906 \mathrm{G}>\mathrm{C}$ & Missense & p.(Ala636Pro) & Class 5 & \\
\hline 260 & MSH2 & c. $1906 \mathrm{G}>\mathrm{C}$ & Missense & p.(Ala636Pro) & Class 5 & \\
\hline 261 & MSH2 & c. $1906 \mathrm{G}>\mathrm{C}$ & Missense & p.(Ala636Pro) & Class 5 & \\
\hline 262 & $M S H 2$ & c. $1943 \mathrm{~T}>\mathrm{A}$ & Missense & p.(Ile648Asn) & & \\
\hline 263 & MSH2 & c.1982_1985del & Frameshift & p.(Lys661Argfs*23) & Class 5 & \\
\hline 264 & MSH2 & c.1986_1987del & Frameshift & p.(Gln662Hisfs*13) & Class 5 & \\
\hline 265 & $M S H 2$ & c.1986del & Frameshift & p.(Met663Cysfs*22) & Class 5 & (27) \\
\hline 266 & MSH2 & c.2006-?_2634+?del & Deletion exons 13-15 & & (Class 5) & (26) \\
\hline 267 & MSH2 & c. $2038 \mathrm{C}>\mathrm{T}$ & Nonsense & p.(Arg680*) & Class 5 & (26) \\
\hline 268 & MSH2 & c. $2038 \mathrm{C}>\mathrm{T}$ & Nonsense & p. $(\operatorname{Arg} 680 *)$ & Class 5 & \\
\hline 269 & $M S H 2$ & c. $2131 \mathrm{C}>\mathrm{T}$ & Nonsense & p.(Arg711*) & Class 5 & (26) \\
\hline 270 & MSH2 & c. $2131 \mathrm{C}>\mathrm{T}$ & Nonsense & p.(Arg711*) & Class 5 & \\
\hline 271 & MSH2 & c. $2164 \mathrm{G}>\mathrm{A}$ & Missense & p.(Val722Ile) & Not classified & \\
\hline 272 & MSH2 & c.2228_2231del & Nonsense & p.(Ser743*) & Class 5 & (26) \\
\hline 273 & MSH2 & c.2234_2236del & Other & p.(Ile747del) & & \\
\hline 274 & $M S H 2$ & c.2234_2236del & Other & p.(Ile747del) & & \\
\hline 275 & MSH2 & c. $2275 \mathrm{G}>\mathrm{T}$ & Nonsense & p.(Gly759*) & Class 5 & \\
\hline 276 & MSH2 & c. $2420 \mathrm{C}>\mathrm{G}$ & Missense & p.(Thr807Ser) & Class 3 & \\
\hline 277 & MSH2 & c. $2635-1 G>A$ & Aberrant splicing & p.(Gln879Valfs*12) & Class 4 & \\
\hline 278 & MSH2 & c. $2635-1 \mathrm{G}>\mathrm{A}$ & Aberrant splicing & p.(Gln879Valfs*12) & Class 4 & \\
\hline 279 & $M S H 2$ & c.2680dup & Frameshift & p.(Met894Asnfs*5) & & \\
\hline 280 & $M S H 2$ & c.2680dup & Frameshift & p.(Met894Asnfs*5) & & \\
\hline 281 & EPCAM & c.185-?_945+?del & Deletion exons 3-9 & & & \\
\hline 282 & MSH6 & c.261-?_457+?dup & Duplication exon 2 & & Class 3 & \\
\hline 283 & MSH6 & c. $463 \mathrm{~A}>\mathrm{G}$ & Missense & p.(Lys155Glu) & & \\
\hline $284^{*}$ & MSH6 & c.773T $>C$ & Missense & p.(Ile258Thr) & & \\
\hline 285 & MSH6 & c.900dup & Frameshift & p.(Lys301Glufs*11) & (Class 5) & \\
\hline 286 & MSH6 & c. $1346 \mathrm{~T}>\mathrm{C}$ & Missense & p.(Leu449Pro) & Class 5 & (27) \\
\hline 287 & MSH6 & c. $1346 \mathrm{~T}>\mathrm{C}$ & Missense & p.(Leu449Pro) & Class 5 & \\
\hline 288 & MSH6 & c. $1346 \mathrm{~T}>\mathrm{C}$ & Missense & p.(Leu449Pro) & Class 5 & \\
\hline 289 & MSH6 & c. $1346 \mathrm{~T}>\mathrm{C}$ & Missense & p.(Leu449Pro) & Class 5 & \\
\hline 290 & MSH6 & c. $1407 \mathrm{~T}>\mathrm{A}$ & Nonsense & p.(Tyr469*) & (Class 5) & \\
\hline 291 & MSH6 & c. $1444 \mathrm{C}>\mathrm{T}$ & Nonsense & p.(Arg482*) & Class 5 & \\
\hline 292 & MSH6 & c. $1483 \mathrm{C}>\mathrm{T}$ & Nonsense & p.(Arg495*) & Class 5 & \\
\hline 293 & MSH6 & c.1499dup & Frameshift & p.(His501Thrfs*6) & (Class 5) & \\
\hline $294 * *$ & MSH6 & c.1649del & Frameshift & p.(Ser550Leufs*21) & (Class 5) & \\
\hline 295 & MSH6 & c. $1691 \mathrm{C}>\mathrm{G}$ & Nonsense & p.(Ser564*) & (Class 5) & \\
\hline 296 & MSH6 & c. $1691 \mathrm{C}>\mathrm{G}$ & Nonsense & p.(Ser564*) & (Class 5) & \\
\hline 297 & MSH6 & c. $1857 \mathrm{~A}>\mathrm{C}$ & Missense & p.(Glu619Asp) & Class 3 & \\
\hline 298 & MSH6 & c.1943del & Frameshift & p.(Ser648Metfs*6) & (Class 5) & \\
\hline 299 & MSH6 & c.2062_2063del & Frameshift & p.(Val688Leufs*9) & Class 5 & \\
\hline 300 & MSH6 & c. $2194 \mathrm{C}>\mathrm{T}$ & Nonsense & p. $(\operatorname{Arg} 732 *)$ & Class 5 & \\
\hline 301 & MSH6 & c. $2299 \mathrm{~A}>\mathrm{T}$ & Missense & p.(Thr767Ser) & & \\
\hline 302 & MSH6 & c.2302_2304del & Other & p.(Pro768del) & Class 3 & (26) \\
\hline 303 & MSH6 & c.2302_2304del & Other & p.(Pro768del) & Class 3 & \\
\hline 304 & MSH6 & c.2302_2304del & Other & p.(Pro768del) & Class 3 & \\
\hline 305 & MSH6 & c. $2608 \mathrm{~A}>\mathrm{G}$ & Missense & p.(Lys870Glu) & & \\
\hline
\end{tabular}


Table I. Continued.

\begin{tabular}{|c|c|c|c|c|c|c|}
\hline $\begin{array}{l}\text { Variant } \\
\text { no. }\end{array}$ & Gene & $\begin{array}{l}\text { Sequence } \\
\text { variant }\end{array}$ & $\begin{array}{l}\text { Type of variant/ } \\
\text { comment }\end{array}$ & $\begin{array}{l}\text { Change at } \\
\text { protein level }\end{array}$ & $\begin{array}{c}\text { InSiGHT } \\
\text { classification }\end{array}$ & Refs. \\
\hline 306 & MSH6 & c. $2732 \mathrm{G}>\mathrm{A}$ & Missense & p.(Arg911Gln) & & \\
\hline 307 & MSH6 & c. $2732 \mathrm{G}>\mathrm{A}$ & Missense & p.(Arg911Gln) & & \\
\hline 308 & MSH6 & c.2779dup & Frameshift & p.(Ile927Asnfs*8) & (Class 5) & \\
\hline 309 & MSH6 & c.2779dup & Frameshift & p.(Ile927Asnfs*8) & (Class 5) & \\
\hline 310 & MSH6 & c.2780_2781insA & Frameshift & p.(Thr928Tyrfs*7) & (Class 5) & \\
\hline 311 & MSH6 & c.2780_2781insA & Frameshift & p.(Thr928Tyrfs*7) & (Class 5) & \\
\hline 312 & MSH6 & c.2780_2781insA & Frameshift & p.(Thr928Tyrfs*7) & (Class 5) & \\
\hline 313 & MSH6 & c.2780_2781insA & Frameshift & p.(Thr928Tyrfs*7) & (Class 5) & \\
\hline 314 & MSH6 & c.2851_2858del & Frameshift & p.(Leu951Ilefs*12) & Class 5 & (26) \\
\hline 315 & MSH6 & c.2851_2858del & Frameshift & p.(Leu951Ilefs*12) & Class 5 & \\
\hline 316 & MSH6 & c. $2931 \mathrm{C}>\mathrm{G}$ & Nonsense & p.(Tyr977*) & Class 5 & (27) \\
\hline 317 & MSH6 & c. $2931 \mathrm{C}>\mathrm{G}$ & Nonsense & p.(Tyr977*) & Class 5 & (27) \\
\hline 318 & MSH6 & c. $2931 \mathrm{C}>\mathrm{G}$ & Nonsense & p.(Tyr977*) & Class 5 & \\
\hline 319 & MSH6 & c. $2931 \mathrm{C}>\mathrm{G}$ & Nonsense & p. $($ Tyr977*) & Class 5 & \\
\hline 320 & MSH6 & c. $2962 \mathrm{C}>\mathrm{T}$ & Missense & p.(Arg988Cys) & & \\
\hline 321 & MSH6 & c.3053_3054del & Frameshift & p.(Leu1018Hisfs*4) & Class 5 & (26) \\
\hline 322 & MSH6 & c. $3103 \mathrm{C}>\mathrm{T}$ & Nonsense & p. $\left(\operatorname{Arg} 1035^{*}\right)$ & Class 5 & \\
\hline 323 & MSH6 & c.3173-?_3556+?del & Deletion exons 5-6 & & (Class 5) & \\
\hline 324 & MSH6 & c.3195_3199del & Frameshift & p.(Asn1065Lysfs*5) & (Class 5) & \\
\hline 325 & MSH6 & c. $3226 \mathrm{C}>\mathrm{T}$ & Missense & p.(Arg1076Cys) & Class 3 & \\
\hline 326 & MSH6 & c.3261del & Frameshift & p.(Phe1088Serfs*2) & Class 5 & \\
\hline 327 & MSH6 & c.3261del & Frameshift & p.(Phe1088Serfs*2) & Class 5 & \\
\hline 328 & MSH6 & c.3261del & Frameshift & p.(Phe1088Serfs*2) & Class 5 & \\
\hline 329 & MSH6 & c.3261dup & Frameshift & p.(Phe1088Leufs*5) & Class 5 & \\
\hline 330 & MSH6 & c.3268_3274del & Frameshift & p.(Glu1090Lysfs*23) & Class 5 & \\
\hline 331 & MSH6 & c. $3299 \mathrm{C}>\mathrm{G}$ & Missense & p.(Thr1100Arg) & & \\
\hline 332 & MSH6 & c.3312del & Frameshift & p.(Phe1104Leufs*11) & Class 5 & \\
\hline 333 & MSH6 & c. $3554 \_3556+2 \mathrm{del}$ & Other & p.(Ser1185_Gly1186de & Cys) & \\
\hline 334 & MSH6 & c.3554_3556+2del & Other & p.(Ser1185_Gly1186de & Cys) & \\
\hline 335 & MSH6 & c.3619_3620del & Frameshift & p.(His1207Phefs*7) & (Class 5) & \\
\hline 336 & MSH6 & c. $3647-2 \mathrm{~A}>\mathrm{C}$ & Aberrant splicing & p.(Arg1217Lysfs*13) & Class 5 & \\
\hline 337 & MSH6 & c. $3674 \mathrm{C}>\mathrm{T}$ & Missense & p.(Thr1225Met) & Class 3 & (26) \\
\hline 338 & MSH6 & c. $3674 \mathrm{C}>\mathrm{T}$ & Missense & p.(Thr1225Met) & Class 3 & \\
\hline 339 & MSH6 & c. $3801+1 \mathrm{del}$ & Aberrant splicing & & & \\
\hline 340 & MSH6 & c.3848_3850dup & Other & p.(Ile1283dup) & & \\
\hline 341 & MSH6 & c.3848_3850dup & Other & p.(Ile1283dup) & & \\
\hline 342 & MSH6 & c. $3878 \mathrm{C}>\mathrm{G}$ & Missense & p.(Ala1293Gly) & & \\
\hline 343 & MSH6 & c.3974_3983dup & Frameshift & p.(Ser1329Aspfs*15) & (Class 5) & \\
\hline 344 & MSH6 & c.3974_3983dup & Frameshift & p.(Ser1329Aspfs*15) & (Class 5) & \\
\hline 345 & MSH6 & c. $3991 \mathrm{C}>\mathrm{T}$ & Aberrant splicing & p.(Ala1268Glyfs*6) & Class 5 & \\
\hline 346 & MSH6 & c. $3991 \mathrm{C}>\mathrm{T}$ & Aberrant splicing & p.(Ala1268Glyfs*6) & Class 5 & \\
\hline 347 & MSH6 & c. $4001+2 \mathrm{~T}>\mathrm{C}$ & Aberrant splicing & p.(Ala1268Glyfs*6) & Class 5 & \\
\hline 348 & MSH6 & c. $4001 \mathrm{G}>\mathrm{A}$ & Missense & p.(Arg1334Gln) & Class 5 & \\
\hline 349 & $P M S 2$ & c.1-?_2586+?del & Whole gene deletion & & Class 5 & \\
\hline 350 & $P M S 2$ & c.24-?_988+?del & Deletion exons 2-9 & & (Class 5) & \\
\hline 351 & PMS2 & c.24-?_988+?del & Deletion exons 2-9 & & (Class 5) & \\
\hline 352 & $P M S 2$ & c.24-?_988+?del & Deletion exons 2-9 & & (Class 5) & \\
\hline 353 & $P M S 2$ & c.24-?_988+?del & Deletion exons 2-9 & & (Class 5) & \\
\hline 354 & $P M S 2$ & c.686_687del & Frameshift & p.(Ser229Cysfs*19) & (Class 5) & \\
\hline 355 & $P M S 2$ & c.736_741delins11 & Frameshift & p.(Pro246Cysfs*3) & Class 5 & $(26)$ \\
\hline 356 & $P M S 2$ & c.736_741delins11 & Frameshift & p.(Pro246Cysfs*3) & Class 5 & (26) \\
\hline
\end{tabular}


Table I. Continued.

\begin{tabular}{|c|c|c|c|c|c|c|}
\hline $\begin{array}{l}\text { Variant } \\
\text { no. }\end{array}$ & Gene & $\begin{array}{l}\text { Sequence } \\
\text { variant }\end{array}$ & $\begin{array}{l}\text { Type of variant/ } \\
\text { comment }\end{array}$ & $\begin{array}{l}\text { Change at } \\
\text { protein level }\end{array}$ & $\begin{array}{c}\text { InSiGHT } \\
\text { classification }\end{array}$ & Refs. \\
\hline 357 & $P M S 2$ & c.736_741delins11 & Frameshift & p.(Pro246Cysfs*3) & Class 5 & \\
\hline 358 & PMS2 & c.736_741delins11 & Frameshift & p.(Pro246Cysfs*3) & Class 5 & \\
\hline 359 & $P M S 2$ & c. $1437 \mathrm{C}>\mathrm{G}$ & Missense & p.(His479Gln) & Class 3 & \\
\hline 360 & PMS2 & c. $1556 \mathrm{~A}>\mathrm{G}$ & Missense & p.(Tyr519Cys) & & \\
\hline 361 & PMS2 & c. $1559 \mathrm{C}>\mathrm{T}$ & Missense & p.(Ala520Val) & & \\
\hline 362 & $P M S 2$ & c. $2113 \mathrm{G}>\mathrm{A}$ & Missense & p.(Glu705Lys) & Class 3 & \\
\hline 363 & $P M S 2$ & c. $2113 \mathrm{G}>\mathrm{A}$ & Missense & p.(Glu705Lys) & Class 3 & \\
\hline 364 & PMS2 & c. $2113 \mathrm{G}>\mathrm{A}$ & Missense & p.(Glu705Lys) & Class 3 & \\
\hline 365 & $P M S 2$ & c. $2113 \mathrm{G}>\mathrm{A}$ & Missense & p.(Glu705Lys) & Class 3 & (26) \\
\hline 366 & PMS2 & c. $2113 \mathrm{G}>\mathrm{A}$ & Missense & p.(Glu705Lys) & Class 3 & \\
\hline 367 & $P M S 2$ & c. $2113 \mathrm{G}>\mathrm{A}$ & Missense & p.(Glu705Lys) & Class 3 & \\
\hline 368 & $P M S 2$ & c. $2113 \mathrm{G}>\mathrm{A}$ & Missense & p.(Glu705Lys) & Class 3 & \\
\hline 369 & $P M S 2$ & c.2520dup & Frameshift & p.(Trp841Leufs*47) & (Class 5) & \\
\hline
\end{tabular}

Variants marked with $*$ and $* *$ represent variants detected in one individual respectively. Classifications made by the authors are listed in parentheses.
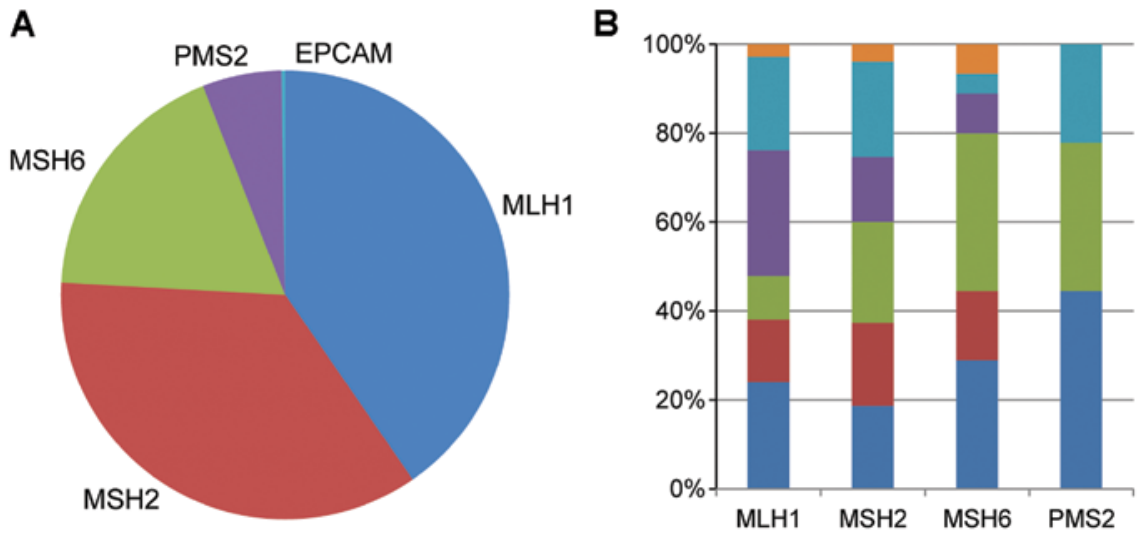

Figure 1. (A) Schematic view of the distribution of the total number of sequence variants in the $M L H 1, M S H 2, M S H 6, P M S 2$ and $E P C A M$ genes from a total of 369 families. (B) Percentage of different sequence variants observed in the MLH1, MSH2, MSH6 and PMS2 genes from a total of 201 variants. The single variant detected in the EPCAM gene is not included. The different changes at the amino acid level are shown in different colors: missense variations are shown in blue, nonsense in red, frameshift variants in green, variants affecting splicing in lilac, whole exon deletions/duplications in turquoise and other changes in orange.

(c.1-?_1076+?del), deletion of exons 1-8 (c.1-?_1386+?del), c.942+3A>T, deletion of exons 7-10 (c.1077-?_1661+?del) and the c.1786_1788del. These variants have previously been reported in Lynch syndrome families from different countries and are classified as disease-predisposing. The deletion of $M S H 2$ exons 1-6 was the most common recurrent variant identified in a total of 10 families. MSH6 had a high number of private mutations with only the c.1346T $>$ C, c. $2780_{-}$ 81insA and the c.2931 $>$ G pathological variants identified in $\geq 4$ families. In $P M S 2$, the sequence variant of unknown significance c. $2113 \mathrm{G}>\mathrm{A}$ was the most common variant found in 7/21 families. The deletions of exons 2-9 (c.24-?_988+?del) and c.736_741delins11 were both identified in 4 families. All of the recurrent sequence variants in MHS6 and PMS2 have previously been reported.
No recurrent mutation suggestive of a Swedish founder mutation was identified. We did, however, recognize a contribution from other Scandinavian founder mutations in the Swedish population. The Finnish founder mutation MLH1 c.1732-?_1896+?del was found in 6\% of the Swedish Lynch syndrome families and constituted $15 \%$ of the MLH1 families. The Danish founder mutation $M L H 1$ c.1667+2_1667+8delinsATTT was observed in two families.

\section{Discussion}

This study is the first compiled data on the Swedish Lynch syndrome cohort and demonstrates mutations in $\mathrm{MLH}$ in $40 \%, M S H 2$ in $36 \%$, MSH6 in $18 \%$ and PMS2 in $6 \%$ of the families (Fig. 1A). The Swedish mutation spectrum is 
Table II. List of mutations occurring four or more times in Swedish families with Lynch syndrome.

\begin{tabular}{|c|c|c|c|c|}
\hline Gene & DNA variant & Protein effect & No. of families & InSiGHT class \\
\hline \multirow[t]{7}{*}{$M L H 1$} & c. $62 \mathrm{C}>\mathrm{T}$ & p.(Ala21 Val) & 4 & 4 \\
\hline & c. $131 \mathrm{C}>\mathrm{T}$ & p.(Ser44Phe) & 5 & 5 \\
\hline & c.454-?_545+? del & p.(Glu153Phefs*8) & 4 & 5 \\
\hline & c. $546-2 \mathrm{~A}>\mathrm{G}$ & p.(Arg182Serfs*6) & 12 & 5 \\
\hline & c.855-?_1038+?del & p.(Ser295Argfs*21) & 4 & 5 \\
\hline & c.1732-?_1896+?del & p.(Pro579_Glu633 del) & 23 & 5 \\
\hline & c. $2059 \mathrm{C}>\mathrm{T}$ & p.(Arg687Trp) & 10 & 5 \\
\hline \multirow[t]{5}{*}{ MSH2 } & c.1-?_1076+?del & p.? & 10 & 5 \\
\hline & c.1?_1386+?del & p.? & 7 & 5 \\
\hline & c. $942+3 \mathrm{~A}>\mathrm{T}$ & p.(Val265_Gln314del) & 7 & 5 \\
\hline & c.1077-?_1661+?del & p.(Arg359_Asn553del) & 6 & 5 \\
\hline & c.1786_1788del & p.(Asn596del) & 6 & 5 \\
\hline \multirow[t]{3}{*}{ MSH6 } & c. $1346 \mathrm{~T}>\mathrm{C}$ & p.(Leu449Pro) & 4 & 5 \\
\hline & c.2780_2781insA & p.(Thr928Tyrfs*7) & 4 & 5 \\
\hline & c. $2931 \mathrm{C}>\mathrm{G}$ & p.(Tyr977*) & 4 & 5 \\
\hline \multirow[t]{3}{*}{$P M S 2$} & c.24-?_988+?del & p.? & 4 & - \\
\hline & c.736_741delins 11 & p.(Pro246Cysfs*3) & 4 & 5 \\
\hline & c. $2113 \mathrm{G}>\mathrm{A}$ & p.(Glu795Lys) & 7 & 3 \\
\hline
\end{tabular}

broad with a total of 201 different mutations of which $66 \%$ were private and $9 \%$ were classified as recurrent, i.e., found in $\geq 4$ families (Table II). The contribution from the different MMR genes is in line with international reports, which are mainly based on Western populations (4). The predominant types of alterations in $M S H 2$ and MSH6 were small insertions/deletions and in $M L H 1$ splice site variants (Fig. 1B). Whole-exon deletions significantly contributed and accounted for $20-22 \%$ of the mutations in $M L H 1, M S H 2$ and $P M S 2$, but were rare (4\%) in MSH6 (Table I, Fig. 1B). Our data support evidence on a significant contribution from whole-exon deletions in $M S H 2$ and $P M S 2$, and demonstrate a higher rate of large deletions than previously reported in $M L H 1(28,29)$. Of the 201 sequence variants reported, 137 are available in the InSiGHT database, whereas 64 have not previously been reported.

In Sweden, $80 \%$ of the population is of Swedish origin and $20 \%$ were either born in another country or born in Sweden by two parents from another country. Among non-Swedish ethnic groups, Finns represent the largest group and during recent decades Sweden has received immigrants from a large number of countries with particularly large contributions from Denmark, Norway, Germany, Chile, former Yugoslavia, Iran, Irak, Eritrea, Somalia and Syria. Strong founder effects have been reported in Finland where two MLH1 mutations account for $63 \%$ of the families with Lynch syndrome (13). The Finnish founder mutation $M L H 1$ c.1732-?_1896+?del, which leads to deletion of $M L H 1$ exon 16, was identified in $6 \%$ of our Lynch syndrome families and constituted $15 \%$ of the $M L H 1$ families, which is in line with the Finnish ancestry in 5\% of the Swedish population. Two families in Sweden carried the Danish founder mutation $M L H 1$ c. $1667+2 \_1667+8$ delinsATTT (8). Two of the most frequent mutations in the Swedish population, i.e., the $M L H 1$ c.546-2A>G and MSH2 c.1-?_1076+?del (deletion of exons 1-6), have been described as founder mutations in the US (30,31). From the mid 1800's until the early 1920's, 1.5 million Swedes migrated to US and it is therefore plausible that this US founder mutation is of Swedish origin. Regarding the deletion of exons 1-6, the common haplotype found in the US was analyzed in two Swedish samples with the same mutation although the results cannot confirm a common ancestry (30). The $M L H 1$ c. $2059 \mathrm{C}>\mathrm{T}$ pathogenic variant is also common in the Swedish population.

Several recurrent mutations identified in $M S H 2$, e.g. the c.1-?_1076+?del, c.942+3A >T and c.1786_1788del have also been reported from Denmark and in Norway (8). Also several of the MSH6 mutations identified, such as c.1444C $>\mathrm{T}, \mathrm{c} .1483 \mathrm{C}>\mathrm{T}$, c.2302_2304del, c.3647-2A >C, c.3991C >T and c. $4001+2 \mathrm{~T}>\mathrm{C}$ have also been observed in several families from Norway and/or Denmark and these mutations may be of Scandinavian origin. In PMS2 the c.736_741delins11 mutations have been reported from Denmark and Norway and the c.2113G $>$ A transition (class 3 ) has also been identified in families from Norway.

We did not detect any individuals with CMMRD in our cohort. Two families harbored more than one MMR gene variants. Both of these families did fulfill the Amsterdam criteria. One family of Arabic origin had a $M L H 1$ c.1989G >A (class 4) variant that affects splicing and a concomitant MSH6 c.773T $>C$ variant, which has not been reported in the ExAc database. Another family had a MSH6 c.1649del frameshift variant and a concomitant $M S H 2$ c. $1484 \mathrm{C}>\mathrm{T}$ variant of unknown significance according to ClinVar. In these families, the $M S H 6$ c. $773 \mathrm{~T}>\mathrm{C}$ and the $M S H 2$ c. $1484 \mathrm{C}>\mathrm{T}$ variants may represent benign variants.

Identification of individuals with Lynch syndrome is cost effective with significant positive effects on morbidity 
and mortality from colorectal cancer (32). In Sweden, Lynch syndrome diagnostics have traditionally been based on individual or physician suspicion of hereditary cancer in which case families have been referred for genetic counseling followed by genetic diagnostics. In total, 369 Lynch syndrome families have been identified. Assuming a carrier frequency in the lower range $(1 / 1,200)$, at least 8,000 individuals would be estimated to be mutation carriers in the Swedish population of 9.8 million. Though the absolute number of mutation carriers in Sweden is not known, it can be estimated that no more than one-quarter of the mutation carriers have at present been identified. Comparison is also possible with our neighboring country Denmark where Lynch syndrome families are registered on a national basis. Denmark has, relative to the size of the population, identified an additional $60 \%$ of Lynch syndrome families (data not shown).

In summary, the Swedish Lynch syndrome cohort with 369 families carries 201 unique alterations, of which 64 have not been previously reported. The mutation spectrum shows the expected contribution from the different MMR genes, underscores the roles of MSH6 and PMS2, which caused $18 \%$ and $6 \%$ of the mutations in the families, respectively. The cohort reveals a higher contribution from large deletion in $M L H 1$ than previously reported. An overlap with mutations identified in the other Nordic countries is identified and our data suggest that US founder mutations in $\mathrm{MLH1}$ and $\mathrm{MSH} 2$ may be of Scandinavian origin.

\section{Acknowledgements}

Financial support was granted from the Swedish Cancer Society. We would like to thank Pål Møller, Oslo, Norway and Christina Therkildsen at the Danish HNPCC register, in Copenhagen for information on mutation spectra in their respective countries. We would also like to acknowledge Eva Rambech and Inger Malmberg for their excellent technical performance.

\section{References}

1. Carethers JM and Stoffel EM: Lynch syndrome and Lynch syndrome mimics: The growing complex landscape of hereditary colon cancer. World J Gastroenterol 21: 9253-9261, 2015.

2. de la Chapelle A: The incidence of Lynch syndrome. Fam Cancer 4: 233-237, 2005.

3. Sijmons RH and Hofstra RM: Review: Clinical aspects of hereditary DNA Mismatch repair gene mutations. DNA Repair (Amst) 38: 155-162, 2016.

4. Thompson BA, Spurdle AB, Plazzer JP, Greenblatt MS, Akagi K, Al-Mulla F, Bapat B, Bernstein I, Capellá G, den Dunnen JT, et al InSiGHT: Application of a 5-tiered scheme for standardized classification of 2,360 unique mismatch repair gene variants in the InSiGHT locus-specific database. Nat Genet 46: 107-115, 2014.

5. Peltomäki P: Role of DNA mismatch repair defects in the pathogenesis of human cancer. J Clin Oncol 21: 1174-1179, 2003.

6. Miyaki M, Konishi M, Tanaka K, Kikuchi-Yanoshita R, Muraoka M, Yasuno M, Igari T, Koike M, Chiba M and Mori T: Germline mutation of MSH6 as the cause of hereditary nonpolyposis colorectal cancer. Nat Genet 17: 271-272, 1997.

7. Berends MJ, Wu Y, Sijmons RH, Mensink RG, van der Sluis T, Hordijk-Hos JM, de Vries EG, Hollema H, Karrenbeld A, Buys CH, et al: Molecular and clinical characteristics of MSH6 variants: An analysis of 25 index carriers of a germline variant. Am J Hum Genet 70: 26-37, 2002.

8. Nilbert M, Wikman FP, Hansen TV, Krarup HB, Orntoft TF, Nielsen FC, Sunde L, Gerdes AM, Cruger D, Timshel S, et al: Major contribution from recurrent alterations and MSH6 mutations in the Danish Lynch syndrome population. Fam Cancer 8: 75-83, 2009.
9. Senter L, Clendenning M, Sotamaa K, Hampel H, Green J, Potter JD, Lindblom A, Lagerstedt K, Thibodeau SN, Lindor NM, et al: The clinical phenotype of Lynch syndrome due to germ-line PMS2 mutations. Gastroenterology 135: 419-428, 2008.

10. Niessen RC, Hofstra RM, Westers H, Ligtenberg MJ, Kooi K, Jager PO, de Groote ML, Dijkhuizen T, Olderode-Berends MJ, Hollema $\mathrm{H}$, et al: Germline hypermethylation of $M L H 1$ and EPCAM deletions are a frequent cause of Lynch syndrome. Genes Chromosomes Cancer 48: 737-744, 2009.

11. Goel A, Nguyen TP, Leung HC, Nagasaka T, Rhees J, Hotchkiss E, Arnold M, Banerji P, Koi M, Kwok CT, et al: De novo constitutional MLH1 epimutations confer early-onset colorectal cancer in two new sporadic Lynch syndrome cases, with derivation of the epimutation on the paternal allele in one. Int J Cancer 128: 869-878, 2011.

12. Kuiper RP, Vissers LE, Venkatachalam R, Bodmer D, Hoenselaar E, Goossens M, Haufe A, Kamping E, Niessen RC, Hogervorst FB, et al: Recurrence and variability of germline EPCAM deletions in Lynch syndrome. Hum Mutat 32: 407-414, 2011.

13. Nyström-Lahti M, Kristo P, Nicolaides NC, Chang SY, Aaltonen LA, Moisio AL, Järvinen HJ, Mecklin JP, Kinzler KW, Vogelstein B, et al: Founding mutations and Alu-mediated recombination in hereditary colon cancer. Nat Med 1: 1203-1206, 1995.

14. Grindedal EM, Aarset H, Bjørnevoll I, Røyset E, Mæhle L, Stormorken A, Heramb C, Medvik H, Møller P and Sjursen W: The Norwegian PMS2 founder mutation c.989-1G > T shows high penetrance of microsatellite instable cancers with normal immunohistochemistry. Hered Cancer Clin Pract 12: 12, 2014.

15. Foulkes WD, Thiffault I, Gruber SB, Horwitz M, Hamel N, Lee C, Shia J, Markowitz A, Figer A, Friedman E, et al: The founder mutation $M S H 2 * 1906 G \rightarrow C$ is an important cause of hereditary nonpolyposis colorectal cancer in the Ashkenazi Jewish population. Am J Hum Genet 71: 1395-1412, 2002.

16. Sun S, Greenwood CM, Thiffault I, Hamel N, Chong G and Foulkes WD: The HNPCC associated $M S H 2 * 1906 G \rightarrow C$ founder mutation probably originated between $1440 \mathrm{CE}$ and $1715 \mathrm{CE}$ in the Ashkenazi Jewish population. J Med Genet 42: 766-768, 2005.

17. Goldberg Y, Porat RM, Kedar I, Shochat C, Galinsky D, Hamburger T, Hubert A, Strul H, Kariiv R, Ben-Avi L, et al: An Ashkenazi founder mutation in the MSH6 gene leading to HNPCC. Fam Cancer 9: 141-150, 2010.

18. Raskin L, Schwenter F, Freytsis M, Tischkowitz M, Wong N, Chong G, Narod SA, Levine DA, Bogomolniy F, Aronson M, et al: Characterization of two Ashkenazi Jewish founder mutations in MSH6 gene causing Lynch syndrome. Clin Genet 79: 512-522, 2011.

19. Tiwari AK, Roy HK and Lynch HT: Lynch syndrome in the 21st century: Clinical perspectives. QJM 109: 151-158, 2015.

20. Hendriks YM, Wagner A, Morreau H, Menko F, Stormorken A, Quehenberger F, Sandkuijl L, Møller P, Genuardi M, Van Houwelingen $\mathrm{H}$, et al: Cancer risk in hereditary nonpolyposis colorectal cancer due to MSH6 mutations: Impact on counseling and surveillance. Gastroenterology 127: 17-25, 2004.

21. Plaschke J, Engel C, Krüger S, Holinski-Feder E, Pagenstecher C, Mangold E, Moeslein G, Schulmann K, Gebert J, von Knebel Doeberitz M, et al: Lower incidence of colorectal cancer and later age of disease onset in 27 families with pathogenic MSH6 germline mutations compared with families with $M L H 1$ or $M S H 2$ mutations: The German Hereditary Nonpolyposis Colorectal Cancer Consortium. J Clin Oncol 22: 4486-4494, 2004.

22. ten Broeke SW, Brohet RM, Tops CM, van der Klift HM, Velthuizen ME, Bernstein I, Capellá Munar G, Gomez Garcia E, Hoogerbrugge N, Letteboer TG, et al: Lynch syndrome caused by germline PMS 2 mutations: Delineating the cancer risk. J Clin Oncol 33: 319-325, 2015.

23. Stoffel E, Mukherjee B, Raymond VM, Tayob N, Kastrinos F, Sparr J, Wang F, Bandipalliam P, Syngal S and Gruber SB: Calculation of risk of colorectal and endometrial cancer among patients with Lynch syndrome. Gastroenterology 137: 1621-1627, 2009.

24. Moreira L, Balaguer F, Lindor N, de la Chapelle A, Hampel H, Aaltonen LA, Hopper JL, Le Marchand L, Gallinger S, Newcomb PA, et al; EPICOLON Consortium: Identification of Lynch syndrome among patients with colorectal cancer. JAMA 308: 1555-1565, 2012.

25. den Dunnen JT and Antonarakis SE: Mutation nomenclature. Curr Protoc Hum genet 37: 7.13.1-7.13.8., 2003. 
26. Lagerstedt Robinson K, Liu T, Vandrovcova J, Halvarsson B, Clendenning M, Frebourg T, Papadopoulos N, Kinzler KW, Vogelstein B, Peltomäki P, et al: Lynch syndrome (hereditary nonpolyposis colorectal cancer) diagnostics. J Natl Cancer Inst 99: 291-299, 2007.

27. Cederquist K, Emanuelsson M, Göransson I, Holinski-Feder E Müller-Koch Y, Golovleva I and Grönberg H: Mutation analysis of the MLH1, MSH2 and MSH6 genes in patients with double primary cancers of the colorectum and the endometrium: A population-based study in northern Sweden. Int J Cancer 109: 370-376, 2004

28. Charbonnier F, Olschwang S, Wang Q, Boisson C, Martin C, Buisine MP, Puisieux A and Frebourg T: $M S H 2$ in contrast to $M L H 1$ and MSH6 is frequently inactivated by exonic and promoter rearrangements in hereditary nonpolyposis colorectal cancer. Cancer Res 62: 848-853, 2002.

29. Smith MJ, Urquhart JE, Harkness EF, Miles EK, Bowers NL, Byers HJ, Bulman M, Gokhale C, Wallace AJ, Newman WG, et al: The contribution of whole gene deletions and large rearrangements to the mutation spectrum in inherited tumor predisposing syndromes. Hum Mutat 37: 250-256, 2016.
30. Clendenning M, Baze ME, Sun S, Walsh K, Liyanarachchi S, Fix D, Schunemann V, Comeras I, Deacon M, Lynch JF, et al: Origins and prevalence of the American founder mutation of MSH2. Cancer Res 68: 2145-2153, 2008.

31. Tomsic J, Liyanarachchi S, Hampel H, Morak M, Thomas BC, Raymond VM, Chittenden A, Schackert HK, Gruber SB, Syngal S, et al: An American founder mutation in MLH1. Int J Cancer 130: 2088-2095, 2012.

32. Snowsill T, Huxley N, Hoyle M, Jones-Hughes T, Coelho H, Cooper C, Frayling I and Hyde C: A systematic review and economic evaluation of diagnostic strategies for Lynch syndrome. Health Technol Assess 18: 1-406, 2014. 